\title{
Efecto del pH sobre la concentración de nutrientes en cacao (Theobroma cacao L.) en la Amazonia Colombiana
}

\section{Effect of pH on the nutrient concentration in cocoa (Theobroma cacao L.) in the Colombian Amazon}

\author{
Gelber Rosas-Patiño ${ }^{1 *}$; Yina Jazbleidi Puentes-Páramo²; Juan Carlos Menjivar-Flores ${ }^{3}$
}

1Ing. Agroecólogo, M.Sc., Ph.D. Universidad de la Amazonía. Florencia - Caquetá, Colombia; e-mail: g.rosas@udla.edu.co; (D) https://orcid.org/0000-00021044-8103

${ }^{2}$ Ing Agrónomo, Ph.D. Consultor independiente en fertilidad y nutrición de cultivos. Palmira-Valle del Cauca-Colombia; e-mail: yjpuentesp@unal.edu.co, (D) https://orcid.org/0000-0002-3784-9687

${ }^{3}$ Ing Agrónomo, Ph.D. Universidad Nacional de Colombia, Sede Palmira. Palmira - Valle del Cauca, Colombia; e-mail: jcmenjivarf@unal.edu.co; (D) https://orcid.org/0000-0002-0985-7778

*autor de correspondencia: g.rosas@udla.edu.co

Cómo citar: Rosas-Patiño, G.; Puentes-Páramo, Y.J.; Menjivar-Flores, J.C. 2021. Efecto del pH sobre la concentración de nutrientes en cacao (Theobroma cacao L.) en la Amazonia Colombiana. Rev. U.D.C.A Act. \& Div. Cient. 24(1):e1643. http://doi.org/10.31910/rudca. v24.n1.2021.1643

Artículo de acceso abierto publicado por Revista U.D.C.A Actualidad \& Divulgación Científica, bajo una Licencia Creative Commons CC BY-NC 4.0

Publicación oficial de la Universidad de Ciencias Aplicadas y Ambientales U.D.C.A, Institución de Educación Superior Acreditada de Alta Calidad por el Ministerio de Educación Nacional.

Recibido: julio 21 de $2020 \quad$ Aceptado: marzo 29 de $2021 \quad$ Editado por: Ingeborg Zenner de Polanía

\section{RESUMEN}

La acidez del suelo limita la disponibilidad, la absorción y la concentración de nutrientes y el rendimiento del cultivo de cacao. El objetivo fue evaluar el efecto del $\mathrm{pH}$ del suelo sobre la concentración de nutrientes en hoja, cáscara y grano, para cuatro clones de cacao autocompatibles (ICS-1, CCN-51) y autoincompatibles (ICS-39, TSH-565), en el departamento del Caquetá. El diseño experimental consistió en un arreglo factorial con cuatro clones (factor A), cuatro niveles de $\mathrm{pH}$ y fertilidad (factor $\mathrm{B}$ ) y cuatro repeticiones. Los resultados indican diferencias en la concentración de nutrientes por efecto del clon y tratamiento, siendo la acumulación de $\mathrm{N}, \mathrm{P}$ y $\mathrm{Mg}$ en grano $>$ hoja $>$ cáscara, $\mathrm{K}$ en cáscara $>$ hoja $>$ grano, Ca, Fe, Mn, Zn y B en hoja $>$ cáscara $>$ grano, S en cáscara $>$ hoja $>$ grano y $\mathrm{Cu}$ en grano>cáscara $>$ hoja. La concentración de P, Mg y S fue mayor y, a su vez, menor Mn, cuando aumentó el pH. El orden de extracción nutrimental en grano fue $\mathrm{N}>\mathrm{K}>\mathrm{P}>\mathrm{Mg}>\mathrm{S}>\mathrm{Ca}>\mathrm{Fe}>\mathrm{Mn}>\mathrm{Zn}>\mathrm{Cu}>\mathrm{B}$. Con relación a los clones, CCN-51 presentó habilidad para la toma de nutrientes y alcanzar mayores producciones, incluso, en suelos con $\mathrm{pH} \geq 5,5$, lo que sugiere efecto de las condiciones edafoclimáticas y, por lo tanto, la necesidad de evaluar los clones para cada zona de cultivo.

Palabras clave: Propiedades suelo; Absorción sustancia nutritivas; Disponibilidad nutrientes; Análisis tejidos; Suelos ácidos.

\section{ABSTRACT}

The soil acidity limits availability, absorption and concentration of nutrients and yield of the cocoa crop. The objective was to 
evaluate the effect of soil $\mathrm{pH}$ on the concentration of macro and micronutrients in leaf, husk and grain for four cocoa clones, self-compatible (ICS-1, CCN-51) and self-incompatible (ICS-39, TSH-565) in the department of Caquetá. The experimental design consisted in a factorial arrangement with four clones (factor A), four $\mathrm{pH}$ and fertility levels (factor B) and four repetitions. The results indicate differences in the concentration of nutrients due to the effect of the clone and the treatment, being the accumulation of $\mathrm{N}, \mathrm{P}$ and $\mathrm{Mg}$ in grain $>$ leaf $>$ husk, $\mathrm{K}$ in husk $>$ leaf $>$ grain, $\mathrm{Ca}, \mathrm{Fe}$, $\mathrm{Mn}, \mathrm{Zn}$ y B in leaf $>$ husk $>$ grain, $\mathrm{S}$ in husk $>$ leaf $>$ grain and $\mathrm{Cu}$ in grain $>$ husk $>$ leaf. The concentration of $\mathrm{P}, \mathrm{Mg}$ and $\mathrm{S}$ was higher, and in turn $\mathrm{Mn}$ lower, when the $\mathrm{pH}$ increased. The order of nutrient extraction in grain was $\mathrm{N}>\mathrm{K}>\mathrm{P}>\mathrm{Mg}>\mathrm{S}>\mathrm{Ca}>\mathrm{Fe}>\mathrm{Mn}>\mathrm{Zn}>\mathrm{Cu}>\mathrm{B}$. Regarding clones, $\mathrm{CCN}-51$ showed ability to take nutrients and reach higher productions, even in soils with $\mathrm{pH} \geq 5.5$, suggesting effect of edaphoclimatic conditions, and therefore, the need to evaluate the clones for each growing area.

Keywords: Soil properties; Nutrient uptake; Tissue analysis; Nutrient availability; Acid soils.

\section{INTRODUCCIÓN}

El cacao (Theobroma cacao L.) es un cultivo tropical originario de la cuenca Amazónica, abunda en los bosques húmedos de Colombia (González-Orozco et al. 2020), donde predominan suelos ácidos (Quesada et al. 2009). Allí crece el cacao, aunque estas condiciones de acidez $(\mathrm{pH}<5,5)$ disminuyen la disponibilidad y la absorción de nutrientes (Rosas-Patiño et al. 2017) y limita la concentración en los tejidos (Quinteiro et al. 2013), afectando el desarrollo de las plantas y, por consiguiente, su rendimiento.

El encalado aumenta el pH y la fertilidad en los suelos, mejora la relación suelo-planta e incrementa la absorción y concentración de nutrientes en la planta (Schlindwein \& Gianello, 2004); esta concentración de nutrientes en un órgano especifico depende de la interacción entre ambiente, genotipo y manejo del cultivo (Correndo \& García, 2012).

Los análisis de tejidos permiten diagnosticar el contenido y la cantidad de nutrientes que extrae el cultivo (Marrocos et al. 2020a) y su distribución en hoja, cáscara y grano, información de gran relevancia para mitigar el impacto de la excesiva fertilización y hacer un eficiente manejo nutricional.
Existen algunas investigaciones acerca de la concentración de nutrientes en cacao, entre ellos, a nivel internacional, Abreu (1996), Malavolta et al. (1997) y Sodré (2002), en Brasil; Aikpokpodion (2010), en África y, más recientemente, Furcal-Beriguete (2017), en Costa Rica; asimismo, a nivel nacional, Puentes-Páramo et al. (2016a; b), sin embargo, son escasos los reportes relacionados con el efecto de los cambios del pH del suelo sobre la extracción de nutrientes en clones de cacao en la Amazonia colombiana.

El objetivo de este trabajo fue evaluar el efecto del $\mathrm{pH}$ en un suelo ácido (Typic Udorthents), sobre la concentración de nutrientes en hoja, cáscara y grano, para cuatro clones de cacao, dos autocompatibles (ICS-1, CCN-51) y dos autoincompatibles (ICS-39, TSH-565).

\section{MATERIALES Y MÉTODOS}

La investigación, se desarrolló en un Typic Udorthents, localizado en el paisaje de lomerío del municipio de Puerto Rico, Departamento de Caquetá, Colombia. La zona, se encuentra en condiciones de Bosque Húmedo Tropical (Ruíz et al. 2007), a 152'12" Latitud Norte y $75^{\circ} 12^{\prime 2} 4^{\prime \prime}$ Longitud Oeste, a $250 \mathrm{~m}$ s.n.m. La región presenta valores medios anuales de precipitación de $2.960 \mathrm{~mm}$, temperatura de $25,3^{\circ} \mathrm{C}$ y humedad relativa de $84 \%$ (IDEAM, 2017).

Se utilizaron cuatro clones de cacao de uso comercial, dos autocompatibles (ICS-1, CCN-51) y dos autoincompatibles (ICS39, TSH-565), establecidos durante siete años, en el Jardín Clonal de la Asociación Departamental de Productores de Cacao y Especies Maderables del Caquetá (ACAMAFRUT).

Se estableció un diseño factorial con cuatro clones (factor A), cuatro tratamientos definidos por el encalado y la fertilización del suelo (factor B) y cuatro repeticiones. Los tratamientos fueron (Tabla 1): To: testigo o suelo con fertilidad natural; T1: disponibilidad de nutrientes después de 90 días de aplicar $7 \mathrm{t} \mathrm{ha}^{-1}$ de cal dolomita, para llevar el suelo a pH $\geq 5,5$ (Rosas-Patiño et al. 2017); T2: aplicación de N-P-K en To, hasta alcanzar los requerimientos del cultivo (PuentesPáramo et al. 2014) y T3: aplicación de N-P-K en T1, hasta alcanzar los requerimientos del cultivo propuestos por los precitados autores.

Para garantizar el efecto de los tratamientos en las unidades

Tabla 1. Valor de pH y concentración de nutrientes por tratamiento.

\begin{tabular}{|l|c|c|c|c|}
\hline \multirow{2}{*}{ Niveles de fertilidad } & \multirow{2}{*}{$\mathbf{p H}$} & \multicolumn{3}{|c|}{ Nutrientes $\left(\mathbf{k g} \mathbf{h} \mathbf{h a}^{-\mathbf{1}}\right)$} \\
\cline { 3 - 5 } & & $\mathbf{N}$ & $\mathbf{P}_{\mathbf{2}} \mathbf{O}_{\mathbf{5}}$ & $\mathbf{K _ { 2 } \mathbf { O }}$ \\
\hline $\mathrm{T}_{0}($ Testigo $)$ & 4,36 & 11,2 & 10,0 & 60,0 \\
\hline $\mathrm{T}_{1}\left(\mathrm{~T}_{0}+\mathrm{Cal}\right)$ & 5,91 & 15,6 & 22,6 & 43,8 \\
\hline $\mathrm{T}_{2}\left(\mathrm{~T}_{0}+\mathrm{N}-\mathrm{P}-\mathrm{K}\right)$ & 4,36 & 73,0 & 35,2 & 219,7 \\
\hline $\mathrm{T}_{3}\left(\mathrm{~T}_{1}+\mathrm{N}-\mathrm{P}-\mathrm{K}\right)$ & 5,91 & 73,0 & 35,2 & 219,7 \\
\hline
\end{tabular}

$\mathrm{N}$ : nitrógeno; $\mathrm{P}_{2} \mathrm{O}_{5}$ : pentóxido de fósforo; $\mathrm{K}_{2} \mathrm{O}$ : óxido de potasio. 
experimentales, se omitió la fertilización durante un año y se eliminaron los frutos para estandarizar la producción. Posteriormente, la fertilización para cada tratamiento, se fraccionó en dos momentos; uno, al inicio de la época lluviosa, en marzo-abril y, otro, en agosto. La unidad experimental estuvo conformada por cuatro árboles sembrados a $3 \mathrm{mx} 3 \mathrm{~m}$, para una densidad poblacional de 1.111 plantas por hectárea.

Los muestreos foliares y de fruto, se realizaron para el año cacaotero 2016-2017. En cada tratamiento y por cada clon, se colectó, de la parte media de cada árbol en plena floración, la cuarta hoja (en total, 25 hojas). Además, se colectaron siete mazorcas maduras y sanas, para determinar la concentración y extracción de nutrientes, por $1.000 \mathrm{~kg}$ de grano seco. Para estimar la extracción, se tuvo en cuenta el tratamiento con mayor rendimiento obtenido en cada clon, de acuerdo con Rosas-Patiño et al. (2019). Las muestras de hoja, cáscara y grano, se secaron en estufa, a $103^{\circ} \mathrm{C}$, durante 24 horas; se molieron y se enviaron al laboratorio de suelos de Agrosavia (Tibaitatá, Colombia) y se determinó el contenido de nitrógeno $(\mathrm{N})$, por EPA 351,3; de fósforo $(\mathrm{P})$, por espectrofotometría; de potasio $(\mathrm{K})$, calcio (Ca) y magnesio $(\mathrm{Mg})$, por espectrofotometría de emisión de plasma inductivamente acoplado; de azufre (S), por turbidimetría; el hierro $(\mathrm{Fe})$, cobre $(\mathrm{Cu})$, manganeso $(\mathrm{Mn})$ y zinc $(\mathrm{Zn})$, se determinaron por absorción atómica, mientras que el boro (B), por ignición.

Los datos fueros sometidos a un Test de normalidad, mediante la prueba Shapiro-Wilks; las variables con comportamiento normal, se evaluaron mediante análisis de varianza, para determinar el efecto de los tratamientos y las interacciones; el ANAVA, para los datos con comportamiento no paramétrico, fue realizada mediante la prueba Kruskal Wallis. Se realizaron pruebas de comparación de medias (Tukey) y correlaciones de Pearson entre variables, con $\mathrm{p} \leq 0,05$, utilizando el programa InfoStat-Profesional, versión 2020p (Di Rienzo et al. 2020).

\section{RESULTADOS Y DISCUSIÓN}

El análisis de varianza (Tabla 2) evidenció efectos altamente significativos en la interacción clon*tratamiento en todos los tejidos, para $\mathrm{K}$ y micronutrientes, con excepción de $\mathrm{Cu}$, que no presentó diferencias en la concentración foliar. $\mathrm{N}$ presentó efecto $(\mathrm{p}<0,01)$, en cáscara y hoja $(\mathrm{p}<0,05)$ y Ca, en hoja $(\mathrm{p}<0,01)$.

El Mg presentó diferencias $(\mathrm{p}<0,01)$ solo en el factor tratamiento. Por su parte, el factor clon evidenció diferencias altamente significativas para $\mathrm{N}$, en grano y $\mathrm{Ca}$, en cáscara; del mismo modo, los clones presentaron efectos significativos en $\mathrm{P}$ almacenado en grano y $\mathrm{Mg}$, en cáscara. Las concentraciones de $\mathrm{P}$, en hoja y cáscara; Ca y Mg, en grano; $\mathrm{Cu}$, en hoja y $\mathrm{S}$, en todos los tejidos, no se vieron afectadas por ningún factor. Los resultados sugieren un efecto de los cambios de $\mathrm{pH}$ y la disponibilidad de nutrientes en el suelo en la absorción de nutrientes en clones de cacao, como lo reportan Baligar \& Fageria (2005), van Vliet et al. (2015) y Rosas-Patiño et al. (2019).

Concentración de macronutrientes en hoja, cáscara y grano de cacao. En general, se observó mayor concentración N, P y Mg, en grano; K, en cáscara; Ca y S, en hoja. Se evidenció, además, el efecto del clon y las condiciones del suelo en la extracción de nutrientes.

Con relación a la acumulación de nutrientes en el tejido foliar, las mayores cantidades de $\mathrm{N}$ se concentraron en grano, probablemente, debido a la necesidad de los genotipos, para favorecer la producción de la semilla (Nehe et al. 2020), seguido de hoja y cáscara, en menor proporción (Figura 1). Este comportamiento es normal en cacao, cuando las tasas de asimilación de $\mathrm{N}$ son más altas en hojas, se ve afectada la producción de los granos (Marrocos et al. 2020b).

La mayor concentración foliar de $\mathrm{N}$ se presentó en $\mathrm{T}_{0}\left(19,57 \mathrm{~g} \mathrm{~kg}^{-1}\right)$, para ICS-1 (Figura 1a) y en $\mathrm{T}_{2}$, para los demás clones, superando los niveles reportados por van Vliet et al. (2015); probablemente,

Tabla 2. ANAVA de la concentración de nutrientes en hoja $(\mathrm{H})$, cáscara $(\mathrm{C})$ y grano de cacao $(\mathrm{G})$, por efecto del clon, tratamiento e interacción.

\begin{tabular}{|c|c|c|c|c|c|c|c|c|c|c|c|c|}
\hline $\begin{array}{l}\text { Fuente de } \\
\text { variación }\end{array}$ & Tejido & $\mathbf{N}$ & $\mathbf{P}$ & $\mathbf{K}$ & $\mathrm{Ca}$ & $\mathbf{M g}$ & $\mathbf{S}$ & $\mathrm{Fe}$ & $\mathrm{Cu}$ & Mn & $\mathrm{Zn}$ & B \\
\hline \multirow{3}{*}{ Clon } & $\mathrm{H}$ & $* *$ & NS & $* *$ & $* *$ & NS & NS & $* *$ & NS & $* *$ & $* *$ & $* *$ \\
\hline & $\mathrm{C}$ & $*$ & NS & $* *$ & $* *$ & $*$ & NS & $* *$ & $* *$ & $* *$ & $* *$ & $* *$ \\
\hline & $G$ & $* *$ & $*$ & NS & $\mathrm{NS}$ & NS & $\mathrm{NS}$ & $* *$ & $* *$ & $* *$ & $* *$ & $* *$ \\
\hline \multirow{3}{*}{ Tratamiento } & $\mathrm{H}$ & NS & NS & $* *$ & $* *$ & $* *$ & NS & $* *$ & NS & $* *$ & $* *$ & $* *$ \\
\hline & $\mathrm{C}$ & $*$ & NS & $*$ & NS & NS & NS & $* *$ & NS & $* *$ & $* *$ & $* *$ \\
\hline & G & NS & $\mathrm{NS}$ & $* *$ & $\mathrm{NS}$ & NS & $\mathrm{NS}$ & $* *$ & $* *$ & $* *$ & $* *$ & $* *$ \\
\hline \multirow{3}{*}{ Clon*'Trat. } & $\mathrm{H}$ & $*$ & NS & $* *$ & $* *$ & NS & NS & $* *$ & NS & $* *$ & $* *$ & $* *$ \\
\hline & $\mathrm{C}$ & $* *$ & $\mathrm{NS}$ & $* *$ & NS & NS & $\mathrm{NS}$ & $* *$ & $* *$ & $* *$ & $* *$ & $* *$ \\
\hline & G & NS & NS & $* *$ & NS & NS & NS & $* *$ & $* *$ & $* *$ & $* *$ & $* *$ \\
\hline
\end{tabular}

** diferencias altamente significativas $(p<0,01)$; diferencias significativas $(p<0,05)$; NS diferencias no significativas $(p>0,05)$. 

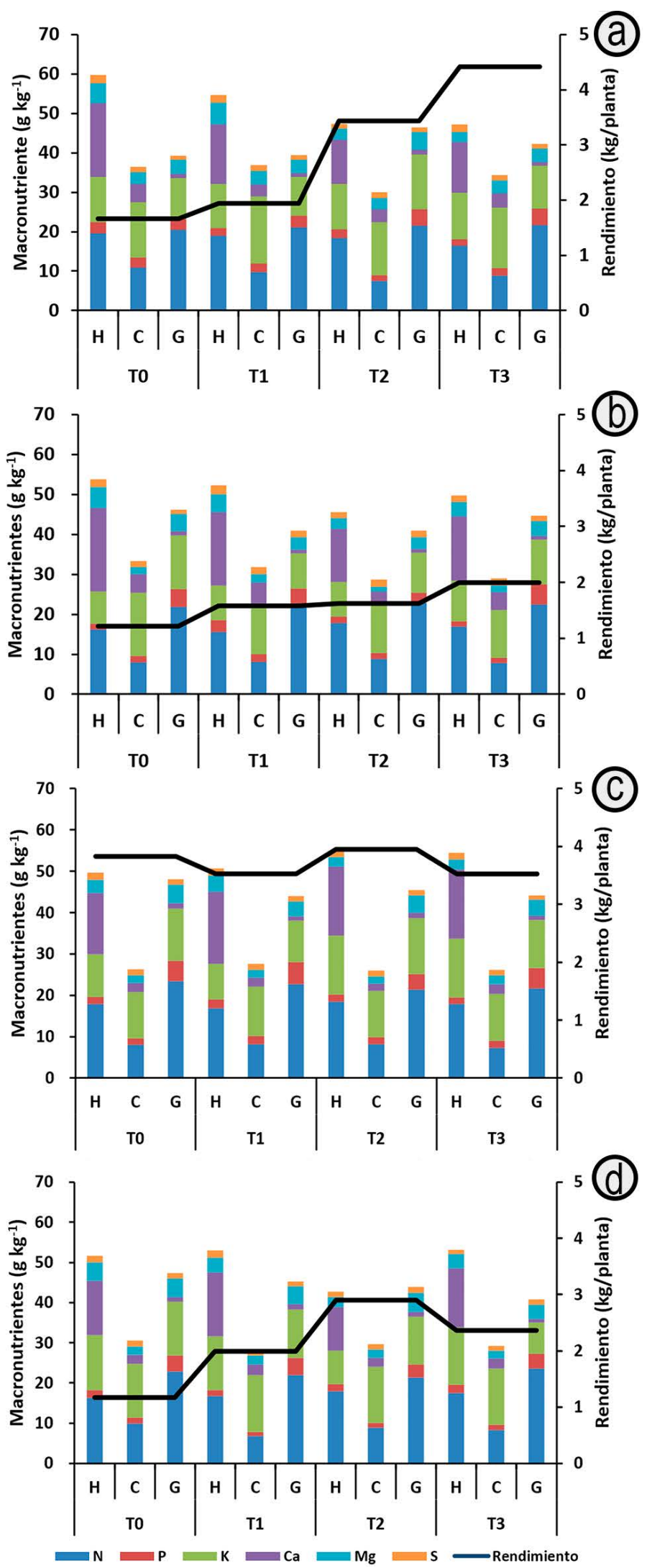

Figura 1. Concentración de macronutrientes en hoja $(\mathrm{H})$, cáscara $(\mathrm{C})$, grano $(\mathrm{G})$ y rendimiento, para cuatro clones de cacao. a. ICS-1; b. ICS-39; c. CCN-51; d. TSH-565. 
las mayores concentraciones $\mathrm{N}$ foliar, en los tratamientos en condiciones de acidez, se debe a las altas concentraciones de Mn, que limitan la redistribución de $\mathrm{N}$ a los demás órganos de la planta (Lavres et al. 2010).

Respecto al P en hoja, el clon ICS-39 (Figura 1b) presentó significativamente el mayor valor en $\mathrm{T}_{1}\left(2,97 \mathrm{~g} \mathrm{~kg}^{-1}\right)$, en donde mejoró la disponibilidad de este elemento en la solución del suelo, producto del encalado, como lo sugieren Anikwe et al. (2016); las mayores concentraciones de $\mathrm{K}$ en hoja, se observaron en el clon CCN-51, para $\mathrm{T}_{2}\left(14,27 \mathrm{~g} \mathrm{~kg}^{-1}\right)$ y en $\mathrm{T}_{3}$, para el resto de clones. El Ca registró el mayor valor en el tejido foliar del clon ICS-39, en $\mathrm{T}_{0}(20,93 \mathrm{~g}$ $\mathrm{kg}^{-1}$ ), dado que, en suelos ácidos, se incrementa la absorción de $\mathrm{Ca}^{2+}$, proporcionalmente a las concentraciones de $\mathrm{H}^{+}$y $\mathrm{Al}^{3+}$, como mecanismo de tolerancia al estrés abiótico (Bojórquez et al. 2014). La mayor extracción de $\mathrm{Mg}$, la presentó ICS-1 (5,57g kg $\left.{ }^{-1}\right)$, en T1, valor considerado normal, dada la mayor disponibilidad y la absorción de este nutriente, en suelos encalados, como lo sugieren Ramírez et al. (2005); la mayor extracción de S (2,23 $\left.\mathrm{g} \mathrm{kg}^{-1}\right)$, la alcanzó ICS-39, en $\mathrm{T}_{1}$. Estos datos son superiores a los reportados por Puentes-Páramo et al. (2016b), quienes evaluaron las extracciones de clones de cacao en zonas de menor temperatura y precipitación media anual y mayor altura sobre el nivel del mar $(1.129 \mathrm{~m}$ más alta); probablemente, los contrastes en las condiciones agroclimáticas de los sitios de estudio resultaron un factor importante en estas diferencias.

En este sentido y tal como se observa en la figura 1, el orden descendente en la concentración de nutrientes foliares para cada clon, en el tratamiento de mayor rendimiento, fue: CCN-51 ( $\left.\mathrm{T}_{1}\right)$ $\mathrm{Ca}>\mathrm{N}>\mathrm{K}>\mathrm{Mg}>\mathrm{P}>\mathrm{S}$, ICS-1 ( $\left.\mathrm{T}_{2}\right)$ y TSH-565 ( $\left.\mathrm{T}_{2}\right) \mathrm{N}>\mathrm{K}>\mathrm{Ca}>$ $\mathrm{Mg}>\mathrm{P}>\mathrm{S}$ e ICS-39 ( $\left.\mathrm{T}_{3}\right) \mathrm{N}>\mathrm{Ca}>\mathrm{K}>\mathrm{Mg}>\mathrm{S}>\mathrm{P}$. En estos tratamientos, las concentraciones de $\mathrm{Mg}, \mathrm{P}$ y $\mathrm{S}$ conservaron el mismo orden en todos los clones, salvo ICS-39, donde la concentración de S fue mayor a $\mathrm{P}$, en contraste con N, K y Ca. Asimismo, se evidenció el efecto de condiciones edáficas sobre el genotipo, especialmente, para CCN-51 (Figura 1c), dado que este clon concentró mayor cantidad de Ca en lugar de N, lo contrario $(\mathrm{N}>\mathrm{Ca}$ ) sugieren Puentes-Páramo et al. (2014). Marschner (2012) afirma que Calcio es un importante mensajero para la respuesta fisiológica de la planta para adaptarse a condiciones ambientales adversas (como la acidez de los suelos), por tanto, es probable que la habilidad de CCN-51 para extraer $\mathrm{Ca}$, corresponda a un mecanismo de respuesta y adaptación del clon a los ambientes tropicales.

La mayor concentración de $\mathrm{N}$ para cáscara se presentó en T $\mathrm{T}_{0}(10,93 \mathrm{~g}$ $\mathrm{kg}^{-1}$ ), en el clon ICS-1 y en T2, para los demás clones, excepto, TSH565 (Figura 1d); este mismo clon presentó la menor concentración en To $\left(9,8 \mathrm{~g} \mathrm{~kg}^{-1}\right)$. Respecto a P, el clon ICS-1 presentó el mayor valor en $\mathrm{T}_{0}\left(2,53 \mathrm{~g} \mathrm{~kg}^{-1}\right)$, el resto de clones en $\mathrm{T}_{1}$, exceptuando el clon TSH-565, que extrajo la menor cantidad $\left(1,43 \mathrm{~g} \mathrm{~kg}^{-1}\right)$ en $\mathrm{T}_{0}$. La mayor concentración de $\mathrm{K}$ en cáscara $\left(17,13 \mathrm{~g} \mathrm{~kg}^{-1}\right)$, se encontró en ICS-1, para $\mathrm{T}_{1}$ y la menor $\left(11,87 \mathrm{~g} \mathrm{~kg}^{-1}\right)$, el clon CCN-51, en este mismo tratamiento.

Con relación al Ca (Figura 1), el mayor valor (4,67 $\left.\mathrm{g} \mathrm{kg}^{-1}\right)$, se registró para ICS-1, en ' $\mathrm{T}_{0}$ y menor para CCN-51, en $\mathrm{T}_{3}\left(2,33 \mathrm{~g} \mathrm{~kg}^{-1}\right)$; la mayor y menor absorción de $\mathrm{Mg}$, se obtuvo en el T1, para el clon ICS-1 (3,40g $\left.\mathrm{kg}^{-1}\right)$ e ICS-39 $\left(2 \mathrm{~g} \mathrm{~kg}^{-1}\right)$, respectivamente y para el S, el T1 del clon ICS-39, reportó el mayor valor $\left(1,80 \mathrm{~g} \mathrm{~kg}^{-1}\right)$. Estas concentraciones de N, P, K, Ca y Mg fueron mayores a las refereridas por FurcalBeriguete (2017). La secuencia de concentración de nutrientes en cáscara en el tratamiento de mayor rendimiento fue (Figura 1): $\mathrm{CCN}-51 \mathrm{~K}>\mathrm{N}>\mathrm{Ca}>\mathrm{P}>\mathrm{Mg}>\mathrm{S}$, ICS-1 $\mathrm{K}>\mathrm{N}>\mathrm{Ca}>\mathrm{Mg}>\mathrm{P}>\mathrm{S}$, TSH-565 $\mathrm{K}>\mathrm{N}>\mathrm{Ca}>\mathrm{Mg}>\mathrm{P}>\mathrm{S}$ e ICS-39 $\mathrm{K}>\mathrm{N}>\mathrm{Ca}>\mathrm{Mg}>\mathrm{P}>\mathrm{S}$; las concentraciones de macronutrientes conservaron el mismo orden, excepto $\mathrm{Mg}$ y P, mientras la mayor concentración en cáscara corresponde a K, como lo reportaron Puentes-Páramo et al. (2014).

La mayor acumulación de $\mathrm{N}$ en grano $\left(23,57 \mathrm{~g} \mathrm{~kg}^{-1}\right)$ la alcanzó el clon TSH-565 (Figura 1d), en T3, consecuente con mayor disponibilidad de nutrientes en este tratamiento, debido al encalado y la fertilización; CCN-51 logró almacenar la mayor cantidad de P $\left(5,47 \mathrm{~g} \mathrm{~kg}^{-1}\right)$, en $\mathrm{T}_{1}$ mientras que las concentraciones de $\mathrm{K}$ más altas $\left(13,9 \mathrm{~g} \mathrm{~kg}^{-1}\right)$, las alcanzó ICS-1, en T3. Con relación a Ca, se registró la mayor concentración $\left(1,33 \mathrm{~g} \mathrm{~kg}^{-1}\right)$ en el clon TSH-565, en $\mathrm{T}_{1}$, mientras que la mayor y menor concentración de $\mathrm{Mg}\left(3,40 \mathrm{~g} \mathrm{~kg}^{-1}\right.$ y $3,07 \mathrm{~g} \mathrm{~kg}^{-1}$ en los clones ICS-1 e ICS-39, respectivamente), se alcanzaron luego del encalado $\left(\mathrm{T}_{1}\right)$; el S, se almacenó en mayor cantidad $\left(1,80 \mathrm{~g} \mathrm{~kg}^{-1}\right)$ en el clon ICS-39, en condiciones de T1 (Figura 1).

Las concentraciones de N, P, K, Ca y Mg fueron mayores comparadas con Furcal-Beriguete (2017). Los clones ICS-1 y TSH565 (Figura 1a;d) mostraron el siguiente orden en la concentración de $\mathrm{N}>\mathrm{K}>\mathrm{Mg}>\mathrm{P}>\mathrm{Ca}>\mathrm{S}$ y los clones ICS-39 y CCN-51, presentaron $\mathrm{N}>\mathrm{K}>\mathrm{P}>\mathrm{Mg}>\mathrm{Ca}>\mathrm{S}$ (Figura 1b;c), encontrando la concentración de $\mathrm{N}$ y S con mayor y menor valor, respectivamente, generalizada para los cuatro clones; además, coincidió con la menor concentración de $\mathrm{S}$, en hoja y en cáscara.

En síntesis, la concentración de $\mathrm{N}$ y $\mathrm{P}$ fue mayor en grano $>$ hoja $>$ cáscara, dado que fósforo se acumula en semillas, como lo propone Marschner (2012); K presentó la mayor concentración en cáscara, seguido hoja y por último grano; Ca fue mayor en hoja>cáscara>grano; $\mathrm{Mg}$ en hoja $>$ grano>cáscara y $\mathrm{S}$ hoja $>$ cáscara $>$ grano. En este orden de ideas, las hojas presentaron mayor concentración de $\mathrm{Ca}, \mathrm{Mg}$ y $\mathrm{S}$, comparadas con la cáscara y el grano; a su vez, la cáscara concentró mayor K y el grano mayor N y P. El clon CCN-51 evidenció mejor comportamiento agronómico puesto que, con valores similares en la concentración de macronutrientes en los tres tejidos, presentó el mayor rendimiento (Figura 1c); así también lo sugieren Cuenca-Cuenca et al. (2019).

\section{Concentración de micronutrientes en hoja, cáscara y grano de} cacao. La concentración de micronutrientes (Fe, Mn, Zn y B) en los tres tejidos (Figura 2), siguió, de manera general, el siguiente orden: hoja $>$ cáscara $>$ grano, correspondiente con el rango de 66-73\%, $15-19 \%$ y $11-15 \%$, respectivamente, salvo $\mathrm{Cu}$, que se concentró, principalmente, en grano, dado que este elemento es esencial para la producción del fruto y la semilla (Marschner, 2012); en este sentido, la mayor concentración de Fe foliar $\left(192,25 \mathrm{mg} \mathrm{kg}^{-1}\right)$, se presentó en TSH-565 (Figura 2d), en $\mathrm{T}_{2}$, tratamiento con alta disponibilidad de cationes ácidos en el suelo, al igual que en el To; $\mathrm{Cu}$, en CCN-51 
(7,81 $\left.\mathrm{mg} \mathrm{kg}^{-1}\right)$, en T3, Mn, en To $\left(1253,45 \mathrm{mg} \mathrm{kg}^{-1}\right)$, para el clon ICS- En el clon ICS-1, para T1 (Figura 2a), se presentó el menor valor de 39, concentración considerada tóxica para cacao (Baligar et al. 2001). $\mathrm{Mn}\left(443,59 \mathrm{mg} \mathrm{kg}^{-1}\right)$. Es probable que el incremento de pH, Ca ${ }^{2+}$ y
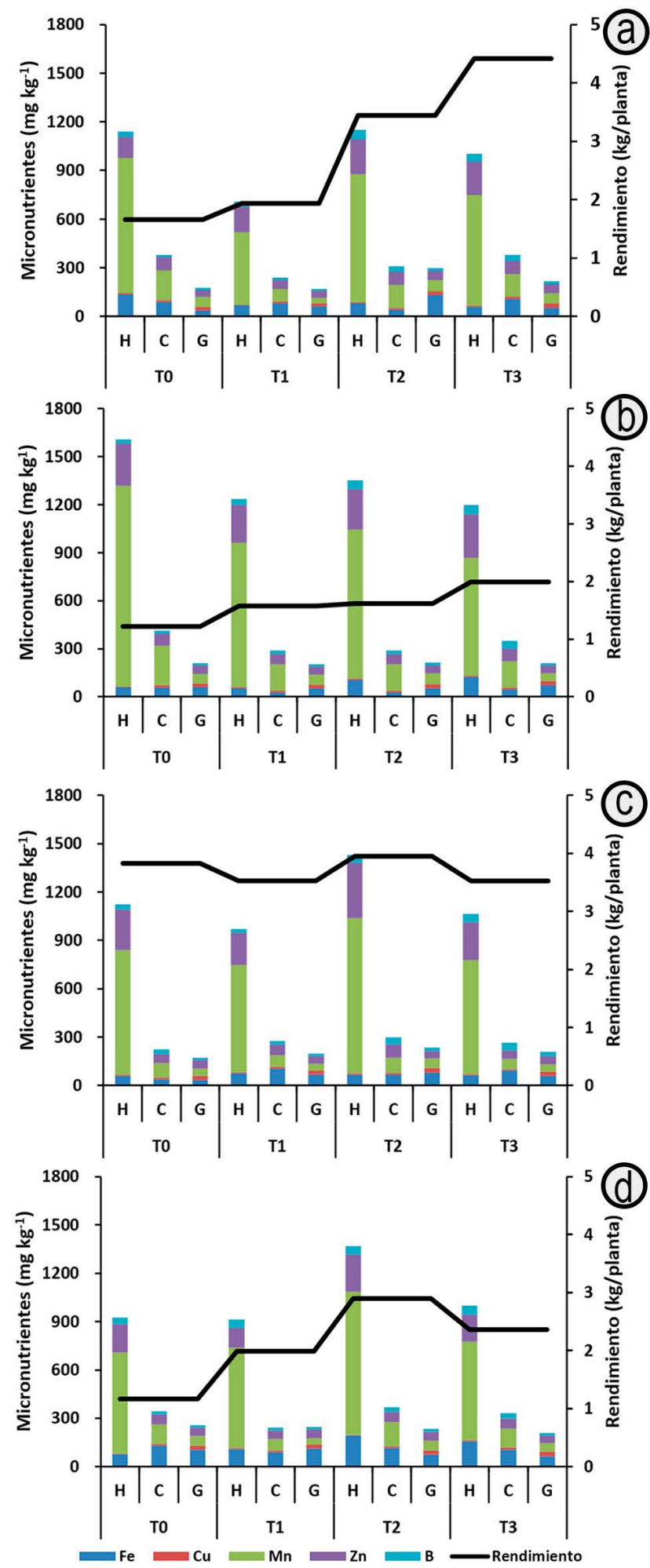

Figura 2. Concentración de micronutrientes en hoja $(\mathrm{H})$, cáscara $(\mathrm{C})$, grano $(\mathrm{G})$ y rendimiento, para cuatro clones de cacao: ICS-1 (A); ICS-39 (B); CCN-51 (C); TSH-565 (D). 
$\mathrm{Mg}$ en el suelo disminuyeran las concentraciones de Mn en los tejidos de cacao (Anda et al. 2013); no obstante, este valor continúa siendo tóxico (Hänsch \& Mendel, 2009). Las cantidades de Zn registradas en CCN-51 (Figura 2c), para T2 $\left(341,18 \mathrm{mg} \mathrm{kg}^{-1}\right)$, son consideradas fitotóxicas, dado que excede el límite de $244 \mathrm{mg} \mathrm{kg}^{-1}$, sugerido por De Oliveira et al. (2015), al igual que B, en T3 $\left(60,26 \mathrm{mg} \mathrm{kg}^{-1}\right)$, para el clon ICS-39 (Figura 2b); comparadas con la referencia de PuentesPáramo et al. (2016b), con relación a otros micronutrientes, las cantidades extraídas se consideran normales para Fe, bajas para $\mathrm{Cu}$ y altas para Mn, Zn y B.

La concentración de micronutrientes, en el tratamiento de mayor rendimiento, fue (Figura 2): $\mathrm{CCN}-51\left(\mathrm{~T}_{1}\right) \mathrm{Fe}>\mathrm{Mn}>\mathrm{Zn}>\mathrm{B}>\mathrm{Cu}$, ICS-1 ( $\left.\mathrm{T}_{2}\right) \mathrm{Mn}>\mathrm{Zn}>\mathrm{Fe}>\mathrm{B}>\mathrm{Cu}$, TSH-565 (T2) $\mathrm{Mn}>\mathrm{Fe}>\mathrm{Zn}>\mathrm{B}>\mathrm{Cu}$ e ICS-39 ( $\left.\mathrm{T}_{3}\right) \mathrm{Mn}>\mathrm{Zn}>\mathrm{B}>\mathrm{Fe}>\mathrm{Cu}$, coincidiendo en presentar la mayor concentración de Fe o Mn, debido a la mayor disponibilidad y absorción de estos nutrientes, favorecida por la acidez del suelo, como lo sugieren Ramírez et al. (2005). Las menores concentraciones fueron para $\mathrm{B}$ y $\mathrm{Cu}$, resultados similares a los obtenidos por PuentesPáramo et al. (2016a).

En cáscara, la mayor concentración de Fe (129,19,25 $\left.\mathrm{mg} \mathrm{kg}^{-1}\right)$, se presentó para el clon TSH-565, en To; Cu, en CCN-51, en $\mathrm{T}_{1}\left(13,65 \mathrm{mg} \mathrm{kg}^{-1}\right)$; Mn, en $\mathrm{T}_{0}\left(250,13 \mathrm{mg} \mathrm{kg}^{-1}\right)$, para el clon ICS39; $\mathrm{Zn}$, en CCN-51, para $\mathrm{T}_{2}\left(82,23 \mathrm{mg} \mathrm{kg}^{-1}\right)$ y $\mathrm{B}$, en $\mathrm{T}_{3}(49,21 \mathrm{mg}$ $\mathrm{kg}^{-1}$ ), clon CCN-51. En orden descendente, la concentración de micronutrientes en el tratamiento con mayor rendimiento fue (Figura 2): $\mathrm{CCN}-51: \mathrm{Fe}>\mathrm{Mn}>\mathrm{Zn}>\mathrm{B}>\mathrm{Cu}$, ICS-1: $\mathrm{Mn}>\mathrm{Zn}>\mathrm{Fe}>\mathrm{B}>\mathrm{Cu}$, TSH-565: $\mathrm{Fe}>\mathrm{Mn}>\mathrm{Zn}>\mathrm{Cu}>\mathrm{B}$ e ICS-39: $\mathrm{Mn}>\mathrm{Zn}>\mathrm{B}>\mathrm{Fe}>\mathrm{Cu}$, coincidiendo en presentar la mayor concentración de un catión ácido, sea Fe o Mn, favorecida por la acidez natural del suelo, como lo sugieren Rosas-Patiño et al. (2017); las menores concentraciones fueron para $\mathrm{B}$ y Cu, resultados similares obtuvo Furcal-Beriguete (2017).

En grano, la mayor concentración de Fe $\left(131,42 \mathrm{mg} \mathrm{kg}^{-1}\right)$, se presentó para ICS-1, en T2; Cu, en TSH-565, en T3 $\left(29,48 \mathrm{mg} \mathrm{kg}^{-1}\right)$; Mn, en $\mathrm{T}_{2}\left(68,56 \mathrm{mg} \mathrm{kg}^{-1}\right)$, en ICS-1; Zn, en TSH-565, para $\mathrm{T}_{2}(55,70 \mathrm{mg}$ $\left.\mathrm{kg}^{-1}\right)$ y B, en $\mathrm{T}_{3}\left(25,37 \mathrm{mg} \mathrm{kg}^{-1}\right)$, clon CCN-51. La concentración de micronutrientes en el tratamiento que presentó el mayor rendimiento fue (Figura 2): clon CCN-51 ( $\left.\mathrm{T}_{1}\right) \mathrm{Fe}>\mathrm{Zn}>\mathrm{Mn}>\mathrm{Cu}>\mathrm{B}$, ICS-1 $\left(\mathrm{T}_{2}\right)$ $\mathrm{Fe}>\mathrm{Mn}>\mathrm{Zn}>\mathrm{B}>\mathrm{Cu}$, TSH-565 (T2) $\mathrm{Fe}>\mathrm{Mn}>\mathrm{Zn}>\mathrm{Cu}>\mathrm{B}$ e ICS39 ( $\left.\mathrm{T}_{3}\right) \mathrm{Fe}>\mathrm{Zn}>\mathrm{Mn}>\mathrm{Cu}>\mathrm{B}$, coincidiendo en presentar la mayor concentración de Fe, favorecida por la acidez del suelo, como lo sugieren Ramírez et al. (2005). Del mismo modo, se presentaron las menores concentraciones de $\mathrm{Cu}$ y $\mathrm{B}$, para todos los clones en estos tratamientos, dado que a $\mathrm{pH}$ superiores a 5,4, se afecta la disponibilidad y absorción (Ambrosini et al. 2015).

La concentración de Fe, Mn, Zn y B en orden descendente para los tres tejidos fue hoja $>$ cáscara $>$ grano, mientras $\mathrm{Cu}$, se almacenó en grano $>$ cáscara $>$ hoja. Lewis et al. (2021) reportaron una correlación positiva entre la disponibilidad de Fe, Mn y Zn en el suelo y las cantidades acumuladas en hojas de cacao. En este orden de ideas, las hojas presentaron mayor concentración de micronutrientes y salvo de $\mathrm{Cu}$; en contraste, el grano concentró mayor Cu y la cáscara presentó la menor concentración de Fe. Además, fue evidente la eficiencia en el uso de nutrientes del clon CCN-51 (Figura 2c), dado que, con menor concentración de micronutrientes en los tejidos, presentó el mayor rendimiento, datos que concuerdan con lo reportado por Cuenca-Cuenca et al. (2019).

\section{Correlación entre la concentración de nutrientes y $\mathrm{pH}$ del} suelo. Las correlaciones entre la concentración de nutrientes en hoja, cáscara y grano de cacao, por efecto del pH (Tabla 3), evidenciaron diferencias entre clones y puede estar relacionado con la diferentes vías de distribución de nutrientes entre hojacáscara-grano de cacao (Quintino et al. 2020); los clones presentan correlaciones positivas para $\mathrm{P}, \mathrm{K}, \mathrm{Mg}, \mathrm{S}, \mathrm{Cu}$ y negativas para $\mathrm{Mn}$ en hoja; para cáscara coincidieron en correlaciones positivas para $\mathrm{P}, \mathrm{Ca}$, $\mathrm{Mg}$, S, B y negativas para Mn y Zn y el grano presentó correlaciones positivas para N, P, Ca, Mg, S, Cu y negativas para K y Mn. De acuerdo con lo anterior, los tres tejidos concentran mayor $\mathrm{P}, \mathrm{Mg}$, $\mathrm{S}$, cuando se eleva el $\mathrm{pH}$ del suelo y cuando es ácido, concentran mayor cantidad de Mn.

Extracción de nutrientes por $1000 \mathrm{~kg}$ de grano seco de cacao. Los clones ICS-1 y TSH-565 coincidieron en las cantidades de macronutrientes extraídos, salvo en los contenidos de $\mathrm{S}$, al igual que se observan las mismas preferencias por $\mathrm{Mn}, \mathrm{Zn}$ y B, pero difieren en la asimilación de Fe y Cu. Por su parte, CCN-51 e ICS-39 extraen similares cantidades de nutrientes, con excepción de $\mathrm{K}$, dado que CCN-51 es el clon que menos cantidades requiere de este elemento (Tabla 4). Los registros de extracción de N, P y K son menores a los reportadas por Quintino et al. (2020), particularmente, las cantidades de $\mathrm{K}$ extraídas por CCN-51. Lo anterior puede corresponder a la habilidad del clon para adaptarse a condiciones de extrema acidez y menor fertilidad natural, sin presentar estrés abiótico (Marschner, 2012).

Las extracciones de micronutrientes (Tabla 4) difieren a las reportadas por Furcal-Beriguete (2017), aunque en otros genotipos, lo cual, evidencia la selectividad en la extracción de micronutrientes entre clones.

Se observó el siguiente orden de extracción de nutrientes en grano (Tabla 4): ICS-1: $\mathrm{N}>\mathrm{K}>\mathrm{Mg}>\mathrm{P}>\mathrm{Ca}=\mathrm{S}>\mathrm{Fe}>\mathrm{Mn}>\mathrm{Zn}>\mathrm{Cu}=\mathrm{B}$; $\mathrm{CCN}-$ 51 e ICS-39: $\mathrm{N}>\mathrm{K}>\mathrm{P}>\mathrm{Mg}>\mathrm{S}>\mathrm{Ca}>\mathrm{Fe}>\mathrm{Zn}>\mathrm{Mn}>\mathrm{Cu}>\mathrm{B}$; TSH-565: $\mathrm{N}>\mathrm{K}>\mathrm{Mg}>\mathrm{P}>\mathrm{S}>\mathrm{Ca}>\mathrm{Fe}>\mathrm{Mn}=\mathrm{Zn}>\mathrm{Cu}>\mathrm{B}$, siendo estos valores y preferencias diferentes y evidenciando el efecto genotipo, como lo sugieren Marrocos et al. (2020b). La secuencia de extracción de los clones, en general, para grano fue: $\mathrm{N}>\mathrm{K}>\mathrm{P}>\mathrm{Mg}>\mathrm{S}>\mathrm{Ca}>\mathrm{Fe}>\mathrm{Mn}-$ $\mathrm{Zn}>\mathrm{Cu}>\mathrm{B}$, ligeramente diferente con los reportes de FurcalBeriguete (2017).

Los resultados obtenidos permiten concluir que el cambio de $\mathrm{pH}$ edáfico y la fertilización afecta la concentración de nutrientes de manera diferencial entre los clones evaluados, a pesar de presentar comportamiento similar en la absorción de nutrientes; CCN-51 alcanza mayor rendimiento de cosecha, incluso, en suelos con $\mathrm{pH}$ extremadamente ácidos. A pesar de las diferencias, los clones presentan las mayores concentraciones de $\mathrm{N}, \mathrm{P}, \mathrm{Mg}$ y $\mathrm{Cu}$ asociadas 
Tabla 3. Correlaciones de Pearson entre la concentración nutrimental en hoja, cáscara, grano seco, para cuatro clones de cacao.

\begin{tabular}{|c|c|c|c|c|c|c|c|c|c|c|c|}
\hline Clon & $\mathbf{N}$ & $\mathbf{P}$ & $\mathrm{K}$ & $\mathrm{Ca}$ & $\mathrm{Mg}$ & $\mathrm{S}$ & $\mathrm{Fe}$ & $\mathrm{Cu}$ & Mn & $\mathrm{Zn}$ & B \\
\hline \multicolumn{12}{|c|}{ Hoja } \\
\hline ICS-1 & $-0,066^{\mathrm{NS}}$ & $0,194^{\mathrm{NS}}$ & $0,561^{\mathrm{NS}}$ & $-0,107^{\mathrm{NS}}$ & $0,253^{\mathrm{NS}}$ & $0,570^{\mathrm{NS}}$ & $-0,642^{*}$ & $0,06^{\mathrm{NS}}$ & $-0,641 *$ & $0,251^{\mathrm{NS}}$ & $-0,131^{\mathrm{NS}}$ \\
\hline ICS-39 & $0,234^{\mathrm{NS}}$ & $0,669^{*}$ & $0,785^{* *}$ & $0,057^{\mathrm{NS}}$ & $0,306^{\mathrm{NS}}$ & $0,570^{\mathrm{NS}}$ & $0,166^{\mathrm{NS}}$ & $0,609 *$ & $-0,645^{*}$ & $-0,115^{\mathrm{NS}}$ & $0,345^{\mathrm{NS}}$ \\
\hline CCN-51 & $0,200^{\mathrm{NS}}$ & $0,604^{*}$ & $0,119^{\mathrm{NS}}$ & $0,863^{* *}$ & $0,558^{\mathrm{NS}}$ & $0,570^{\mathrm{NS}}$ & $0,533^{\mathrm{NS}}$ & $0,820^{* *}$ & $-0,517^{\mathrm{NS}}$ & $-0,469^{\mathrm{NS}}$ & $-0,06^{\mathrm{NS}}$ \\
\hline TSH-565 & $0,557^{\mathrm{NS}}$ & $0,516^{\mathrm{NS}}$ & $0,498^{\mathrm{NS}}$ & $0,738^{* *}$ & $0,372^{\mathrm{NS}}$ & $0,570^{\mathrm{NS}}$ & $0,123^{\mathrm{NS}}$ & $0,528^{\mathrm{NS}}$ & $-0,331^{\mathrm{NS}}$ & $-0,480^{\mathrm{NS}}$ & $0,582^{*}$ \\
\hline \multicolumn{12}{|c|}{ Cáscara } \\
\hline ICS-1 & $0,191^{\mathrm{NS}}$ & $0,500^{\mathrm{NS}}$ & $0,859^{* *}$ & $0,133^{\mathrm{NS}}$ & $0,742^{* *}$ & $0,570^{\mathrm{NS}}$ & $0,373^{\mathrm{NS}}$ & $0,319^{\mathrm{NS}}$ & $-0,589 *$ & $-0,349^{\mathrm{NS}}$ & $0,149^{\mathrm{NS}}$ \\
\hline ICS-39 & $0,371^{\mathrm{NS}}$ & $0,578^{*}$ & $-0,177^{\mathrm{NS}}$ & $0,693^{*}$ & $0,644^{*}$ & $0,570^{\mathrm{NS}}$ & $-0,630^{*}$ & $-0,098^{\mathrm{NS}}$ & $-0,605^{*}$ & $-0,355^{\mathrm{NS}}$ & $0,212^{\mathrm{NS}}$ \\
\hline CCN-51 & $0,365^{\mathrm{NS}}$ & $0,679 *$ & $0,728^{* *}$ & $0,647 *$ & $0,640^{*}$ & $0,570^{\mathrm{NS}}$ & $0,769^{* *}$ & $0,419^{\mathrm{NS}}$ & $-0,663^{* *}$ & $-0,248^{\mathrm{NS}}$ & $0,065^{\mathrm{NS}}$ \\
\hline TSH-565 & $-0,344^{\mathrm{NS}}$ & $0,215^{\mathrm{NS}}$ & $0,549^{* *}$ & $0,141^{\mathrm{NS}}$ & $0,359^{\mathrm{NS}}$ & $0,462^{\mathrm{NS}}$ & $-0,342^{\mathrm{NS}}$ & $0,371^{\mathrm{NS}}$ & $-0,588^{*}$ & $-0,480^{\mathrm{NS}}$ & $0,337^{\mathrm{NS}}$ \\
\hline \multicolumn{12}{|c|}{ Grano } \\
\hline ICS-1 & $0,698^{*}$ & $0,564^{\mathrm{NS}}$ & $-0,031^{\mathrm{NS}}$ & $0,474^{\mathrm{NS}}$ & $0,335^{\mathrm{NS}}$ & $0,570^{\mathrm{NS}}$ & $-0,137^{\mathrm{NS}}$ & $0,374^{\mathrm{NS}}$ & $-0,400^{\mathrm{NS}}$ & $0,191^{\mathrm{NS}}$ & $-0,024^{\mathrm{NS}}$ \\
\hline ICS-39 & $0,654^{*}$ & $0,582^{*}$ & $-0,196^{\mathrm{NS}}$ & $0,531^{\mathrm{NS}}$ & $0,291^{\mathrm{NS}}$ & $0,570^{\mathrm{NS}}$ & $0,303^{\mathrm{NS}}$ & $0,409^{\mathrm{NS}}$ & $-0,304^{\mathrm{NS}}$ & $-0,240^{\mathrm{NS}}$ & $-0,136^{\mathrm{NS}}$ \\
\hline CCN-51 & $0,254^{\mathrm{NS}}$ & $0,690^{*}$ & $-0,246^{\mathrm{NS}}$ & $0,502^{\mathrm{NS}}$ & $0,239^{\mathrm{NS}}$ & $0,570^{\mathrm{NS}}$ & $0,329^{\mathrm{NS}}$ & $0,266^{\mathrm{NS}}$ & $-0,319^{\mathrm{NS}}$ & $0,328^{\mathrm{NS}}$ & $0,263^{\mathrm{NS}}$ \\
\hline TSH-565 & $0,531 *$ & $0,568^{*}$ & $-0,244^{\mathrm{NS}}$ & $0,511 *$ & $0,314^{\mathrm{NS}}$ & $0,561 *$ & $-0,023^{\mathrm{NS}}$ & $0,254^{\mathrm{NS}}$ & $-0,662 * *$ & $-0,066^{\mathrm{NS}}$ & $-0,046^{\mathrm{NS}}$ \\
\hline
\end{tabular}

** Correlación altamente significativa al nivel $0,01, *$ correlación significativa al nivel 0,05 , NS no significativa.

Tabla 4. Extracción de nutrientes en kg por 1.000kg de grano seco, para cuatro clones de cacao.

\begin{tabular}{|l|c|c|c|c|c|c|c|c|c|c|c|}
\hline \multicolumn{1}{|c|}{ Clon } & $\mathbf{N}$ & $\mathbf{P}$ & $\mathbf{K}$ & $\mathbf{C a}$ & $\mathbf{M g}$ & $\mathbf{S}$ & $\mathbf{F e}$ & $\mathbf{C u}$ & $\mathbf{M n}$ & $\mathbf{Z n}$ & $\mathbf{B}$ \\
\hline ICS-1 & $21,53^{\mathrm{a}}$ & $4,20^{\mathrm{ab}}$ & $13,90^{\mathrm{a}}$ & $1,20^{\mathrm{a}}$ & $4,37^{\mathrm{a}}$ & $1,20^{\mathrm{a}}$ & $0,13^{\mathrm{a}}$ & $0,02^{\mathrm{b}}$ & $0,07^{\mathrm{a}}$ & $0,06^{\mathrm{a}}$ & $0,02^{\mathrm{a}}$ \\
\hline CCN-51 & $22,60^{\mathrm{a}}$ & $5,47^{\mathrm{a}}$ & $9,97^{\mathrm{c}}$ & $0,97^{\mathrm{b}}$ & $3,77^{\mathrm{b}}$ & $1,30^{\mathrm{a}}$ & $0,07^{\mathrm{b}}$ & $0,03^{\mathrm{a}}$ & $0,04^{\mathrm{b}}$ & $0,05^{\mathrm{b}}$ & $0,02^{\mathrm{a}}$ \\
\hline ICS-39 & $22,47^{\mathrm{a}}$ & $5,03^{\mathrm{ab}}$ & $11,20^{\mathrm{b}}$ & $0,95^{\mathrm{b}}$ & $3,70^{\mathrm{b}}$ & $1,27^{\mathrm{a}}$ & $0,07^{\mathrm{b}}$ & $0,03^{\mathrm{a}}$ & $0,05^{\mathrm{b}}$ & $0,05^{\mathrm{b}}$ & $0,02^{\mathrm{a}}$ \\
\hline TSH-565 & $21,33^{\mathrm{a}}$ & $3,30^{\mathrm{b}}$ & $11,90^{\mathrm{ab}}$ & $1,20^{\mathrm{a}}$ & $4,67^{\mathrm{a}}$ & $1,47^{\mathrm{b}}$ & $0,08^{\mathrm{b}}$ & $0,03^{\mathrm{a}}$ & $0,06^{\mathrm{a}}$ & $0,06^{\mathrm{a}}$ & $0,02^{\mathrm{a}}$ \\
\hline
\end{tabular}

Valores por columna con letras iguales no difieren en forma significativa $(\mathrm{P}<0,05)$, según prueba de Tukey.

a grano, $\mathrm{Ca}, \mathrm{Fe}, \mathrm{Mn}, \mathrm{Zn}$ y B asociadas a hoja y K, preferiblemente, en cáscara; asimismo, concentran mayor $\mathrm{P}, \mathrm{Mg}, \mathrm{S}$, cuando $\mathrm{pH} \geq 5,5 \mathrm{y}$ mayor Mn, cuando el suelo se encuentra en condiciones de acidez.

Agradecimientos. Los autores expresan gratitud a la Asociación Departamental de Productores de Cacao y Especies Maderables del Caquetá (Acamafrut) y a la Universidad de la Amazonia, por su apoyo a esta investigación. Conflictos de intereses: El manuscrito fue preparado con la participación de todos los autores, quienes declaran que no existe ningún conflicto de intereses que ponga en riesgo la validez de los resultados presentados.

\section{REFERENCIAS}

1. ABREU, J.C. 1996. Foliar nutrient concentration and rations in height yield cocoa genotypes and relations yield and intensity of witches broom disease. In: Costa, R. (eds). Internal Conf. on Cacao Research. Cocoa Producer's Alliance. CEPLAC/CEPEC. Atas, Lagos, NGR. p.773-780.
2. AIKPOKPODION, P.E. 2010. Nutrients dynamics in cocoa soils, leaf and beans in Ondo State, Nigeria. Agri Sci. 1(1):1-9.

https://doi.org/10.1080/09766898.2010.11884647

3. AMBROSINI, V.G.; ROSA, D.J.; CORREDOR, J.P.; BORGHEZAN, M.; BASTOS DE MELO, G.W.; FONSECA DE SOUSA, C.R.; COMIN, J.J.; GUIMARÃES, D.; BRUNETTO, G. 2015. Reduction of copper phytotoxicity by liming: A study of the root anatomy of young vines (Vitis labrusca L.). Plant Physiology and Biochemistry. 96:270-280.

https://doi.org/10.1016/j.plaphy.2015.08.012

4. ANDA, M.; SHAMSHUDDIN, J.; FAUZIAH, C.I. 2013. Increasing negative charge and nutrient contents of a highly weathered soil using basalt and rice husk to promote cocoa growth under field conditions. Soil \& Tillage Research. 132:1-11.

https://doi.org/10.1016/j.still.2013.04.005 
5. ANIKWE, M.A.; EZE, J.C.; IBUDIALO, A.N. 2016. Influence of lime and gypsum application on soil properties and yield of cassava (Manihot esculenta Crantz.) in a degraded Ultisol in Agbani Enugu Southeastern Nigeria. Soil and Tillage Research. 158:32-38. https://doi.org/10.1016/j.still.2015.10.011

6. BALIGAR, V.C; FAGERIA, N.; HE, Z. 2001. Nutrient use efficiency in plants. Communications in Soil Science and Plant Analysis. 32(7):921-950.

7. BALIGAR, V.C.; FAGERIA, N.K. 2005. Aluminum influence on growth and uptake of micronutrients by cacao. J. Food Agriculture and Environment. 3(3-4):173-177.

8. BOJÓRQUEZ, Q.E.; SÁNCHEZ, C.L.; KU, G.A.; De los SANTOS, B.C.; MEDINA, L.F.; ECHEVERRÍA, I.; MÚÑOZ, S.J.; HERNÁNDEZ, S.T.; MARTÍNEZ, E.M. 2014. Differential effects of aluminum on in vitro primary root growth, nutrient content and phospholipase $\mathrm{C}$ activity in coffee seedlings (Coffea arabica L.). J. Inorganic Biochemistry. 134:39-48.

https://doi.org/10.1016/j.jinorgbio.2014.01.018

9. CORRENDO, A.A.; GARCÍA, F.O. 2012. Concentración de nutrientes en planta como herramienta de diagnóstico: Cultivos extensivos. Archivo Agronómico No. 14. Informaciones Agronómicas de Hispanoamérica. No. 5. IPNI Cono Sur. Buenos Aires. Disponible desde Internet en:

http://lacs.ipni.net/article/LACS-1155 (con acceso 9/07/2020).

10. CUENCA-CUENCA, E.W.; PUENTES-PÁRAMO, Y.J.; MENJIVAR FLORES, J.C. 2019. Efficient use of nutrients in fine aroma cacao in the province of Los Ríos-Ecuador. Rev. Fac. Nac. Agron. Medellín. 72(3):8963-8970.

11. DE OLIVEIRA, C.N.R.; DE SOUZA, J.J.; ANDRADE, S.G.; BALIGAR, V.C. 2015. Growth and nutrition of cacao seedlings influenced by zinc aplication in soil. Rev. Bras. Frutic., Jaboticabal. 37(4):1053-1064. https://doi.org/10.1590/0100-2945-238/14

12. DI RIENZO, J.A.; CASANOVES, F.; BALZARINI, M.G.; GONZALEZ, L.; TABLADA, M.; ROBLEDO, C.W. 2020. InfoStat Version 2020. Grupo Centro de transferencia InfoStat, FCA, Universidad Nacional de Córdoba (Argentina). Disponible desde Internet en: http://www.infostat.com.ar (con acceso 9/07/2020).

13. FURCAL BERIGUETE, P. 2017. Extracción de nutrientes por los frutos de cacao en dos localidades en Costa Rica. Agron. Mesoam. 28(1):113-129.

https://doi.org/10.15517/am.v28i1.23236
14. GONZÁLEZ OROZCO, C.E.; SÁNCHEZ GALÁN, A.A.; RAMOS, P.F.; YOCKTENG, R. 2020. Exploring the diversity and distribution of crop wild relatives of cacao (Theobroma cacao L.) in Colombia. Genet Resour Crop Evol. 67:2071-2085.

https://doi.org/10.1007/s10722-020-00960-1

15. HÄNSCH, R.; MENDEL, R.R. 2009. Physiological functions of mineral micronutrients ( $\mathrm{Cu}, \mathrm{Zn}, \mathrm{Mn}, \mathrm{Fe}, \mathrm{Ni}, \mathrm{Mo}, \mathrm{B}, \mathrm{Cl})$. Current Opinion in Plant Biology. 12:259-266. https://doi.org/10.1016/j.pbi.2009.05.006

16. INSTITUTO DE HIDROLOGÍA Y ESTUDIOS AMBIENTALES, IDEAM. 2017. Indicadores climáticos. Disponible desde Internet en: http://institucional.ideam.gov.co/jsp/1772 (con acceso el $7 / 07 / 2020)$

17. LAVRES, J.J.; REIS, A.R.; ROSSI, M.L.; CABRAL, C.P.; NOGUEIRA, N.L.; MALAVOLTA, E. 2010. Changes in the ultrastructure of soybean cultivars in response to manganese supply in solution culture. Sci. Agric. 67:287294.

https://doi.org/10.1590/S0103-90162010000300006

18. LEWIS, C.; LENNON, A.M.; EUDOXIE, G.; SIVAPATHAM, P.; UMAHARAN, P. 2021. Plant metal concentrations in Theobroma cacao as affected by soil metal availability in different soil types. Chemosphere. 262:127749. https://doi.org/10.1016/j.chemosphere.2020.127749

19. MALAVOLTA, E.; VITTI, G.C.; DE OLIVEIRA, S.A. 1997. Avaliação do estado nutricional das plantas. Principios e aplicações. 2a edicicão. Piracicaba. Associa. Bras. para Pesq. da Potassa e do Fosfato. (Brasil). 319p.

20. MARrocos, P.; GUILLERME LOUREIRO, G.A.; QUINTINO, R. DE A.; SODRÉ, A.; AHNERT, D.; BALIGAR, V. 2020a. Mineral nutrient ratios and cacao productivity. J. Plant Nutrition. 43(15):2368-2382. https://doi.org/10.1080/01904167.2020.1771582

21. MARROCOS, P.; LOUREIRO, G.A.; DE ARAUJO, G.; SODRÉ A.; AHNERT, D.; ESCALONA, V.R.; BALIGAR, V. 2020b. Mineral nutrition of cacao (Theobroma cacao L.): relationships between foliar concentrations of mineral nutrients and crop productivity. J. Plant Nutrition. 43(10):1498-1509. https://doi.org/10.1080/01904167.2020.1739295

22. MARSCHNER, P. 2012. Mineral Nutrition of Higher Plants, third ed. Academic Press (New York).

23. NEHE, S.; MMISRA, S.; MURCHIE, H.; CHINNATHAMBI, K.; SINGH TYAGI, B.; FOULKES, J. 2020. Nitrogen 
partitioning and remobilization in relation to leaf senescence, grain yield and protein concentration in Indian wheat cultivars. Field Crops Research.

https://doi.org/10.1016/j.fcr.2020.107778

24. PUENTES-PÁRAMO, Y.J.; GÓMEZ CARABALÍ, C.A.; MENJIVAR FLORES, J.C. 2016a. Influence of the relationship among nutrients on yield of cocoa (Theobroma cacao L.) clones. Act. Agron. 65(2):176-182.

https://doi.org/10.15446/acag.v65n2.47387

25. PUENTES-PÁRAMO, Y.J.; MENJIVAR FLORES, J.C.; ARANZAZU HERNÁNDEZ, F. 2016b. Concentración de nutrientes en hoja, una herramienta para el diagnóstico nutriciones en cacao. Agron. Mesoam. 27(2):329-336. https://doi.org/10.15517/am.v27i2.19728

26. PUENTES-PÁRAMO, Y.J.; MENJIVAR FLORES, J.C.; GÓMEZ CARABALÍ, C.A.; ARANZAZU HERNÁNDEZ, F. 2014. Absorción y distribución de nutrientes en clones de cacao y sus efectos en el rendimiento. Acta Agronómica. 63(2):145-152.

https://doi.org/10.15446/acag.v63n2.40041

27. QUESADA, C.A.; LLOYD, J.; ANDERSON, L.O.; FYLLAS, N.M.; SCHWARZ, M.; CZIMCZIK, C.I. 2009. Soils of Amazonia with particular reference to the rainfor sites. Biogeosciences Discuss. 6:3851-3921. https://doi.org/10.5194/bg-8-1415-2011

28. QUINTEIRO, M.; FURTADO, A.; SCHRAMM, M.; PINTO, F.; VIANA, M.; BALIGAR, V.C. 2013. Aluminum effects on growth photosynthesis and mineral nutrition of cacao genotypes. J. Plant Nutrition. 36(8):1161-1179. https://doi.org/10.1080/01904167.2013.766889

29. QUINTINO, R. DE A.; GUILHERME, A.H. DE A.L.; AHNERT, D.; ESCALONA-VALDEZ, R.A.; BALIGAR, V.C. 2020. Interactions between Soil, Leaves and Beans Nutrient Status and Dry Biomass of Beans and Pod Husk of Forastero Cacao: An Exploratory Study. Communications in Soil Science and Plant Analysis. 51(5):567-581.

https://doi.org/10.1080/00103624.2020.1729369

30. RAMÍREZ, R.V.; LÓPEZ, B.J.; HERRERA, E.L. 2005. Adaptive responses in plants to nonoptimal soil $\mathrm{pH}$. En: Jenks, M.; Hasegawa, P. (eds). Plant abiotic stress. Center for Plant Environmental Stress Physiology Purdue University Indiana, USA. 266p.

31. ROSAS PATIÑO, G.; PUENTES-PÁRAMO, Y.J.; MENJIVAR FLORES, J.C. 2017. Relación entre el pH y la disponibilidad de nutrientes para cacao en un entisol de la Amazonia colombiana. Corpoica Cienc Tec. Agropec (Mosquera, Colombia). 18(3):529-541.

https://doi.org/10.21930/rcta.vol18_num3_art:742

32. ROSAS PATIÑO, G.; PUENTES-PÁRAMO, Y.J.; MENJIVAR FLORES, J.C. 2019. Efecto del encalado en el uso eficiente de macronutrientes para cacao (Theobroma cacao L.) en la Amazonia colombiana. Ciencia Y Tec. Agropec. 20(1):05-16. https://doi.org/10.21930/rcta.vol20_num1_art:1247

33. RUÍZ, J.; CÁRDENAS, W.; BAQUERO, C. 2007. Cambio de la cobertura boscosa como sucedáneo de la dinámica colonizadora en el departamento del Caquetá 1986-2000. Rev. de Inves. Univ de la Salle. 7(1):95-104.

34. SCHLINDWEIN, J.A.; GIANELLO, C. 2004. Necessidades de novos estudos de calibração e recomendação de fertilizantes para as culturas cultivadas sob plantio direto. R. Plantio Direto. 79:12-15.

35. SODRÉ, G.A. 2002. Uso do desvio padrão para estimativa do tamaño de amostra de plantas de cacau (Theobroma cacao L.) em estudos de nutriçao. Agrotrópica. 13:145-150.

36. VAN VLIET, J.A.; SLINGERLAND, M.; GILLER, K.E. 2015. Mineral Nutrition of Cocoa. A Review. Wageningen University and Research Centre, Wageningen. 57p. https://doi.org/10.1016/bs.agron.2016.10.017 